\title{
Orbital Elements of 2004 Perseid Meteoroids Perturbed by Jupiter
}

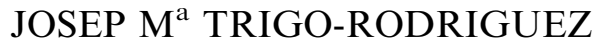 \\ Institut Ciències de l'Espai (CSIC), \\ Campus UAB, Facultat de Ciències, Torre C5-parell-2 ${ }^{a}, 08193$, Bellaterra, Barcelona, Spain and \\ Institut d'Estudis Espacials de Catalunya (IEEC), \\ Edif., Nexus, c/Gran Capità, 2-4, 08034, Barcelona, Spain \\ JEREMIE VAUBAILLON \\ University of Western Ontario, London, Ontario, Canada \\ JOSÉ LUÍS ORTIZ, ALBERTO CASTRO-TIRADO, MARTIN \\ JELÍNEK, ANTONIO DE UGARTE POSTIGO, PABLO SANTOS \\ SANZ, FRANCISCO J. ACEITUNO CASTRO \\ Instituto de Astrofisica de Andalucia (IAA-CSIC), PO Box 3004, 18080, Granada, Spain
}

JORDI LLORCA

Institut de Tècniques Energètiques, Universitat Politècnica de Catalunya,

Diagonal 647, 08028, Barcelona, Spain

ESKO LYYTINEN

Kehakukantie 3 B, 00720 Helsinki, Finland

ALBERT SÁNCHEZ CASO

Gualba Observatory, (MPC 442), Barcelona, Spain

ANTONIO BERNAL GONZÁLEZ

Asociación Astronómica de Castelldefels, Barcelona, Spain

JUAN PASTOR ERADES

Agrupació Astronòmica de Sabadell, Barcelona, Spain

FRANCISCO OCAÑA

Agrupación Astronómica de Madrid, Madrid, Spain

(Received 16 November 2005; Accepted 22 February 2006)

\begin{abstract}
Jupiter and Saturn produce important gravitational impulses on meteoroids released by comet 109P/Swift-Tuttle. The meteoroids from this comet once released follow retrograde orbits that during their periodic approaches to these planets (within 1.6 and 0.9 A.U., respectively) are impulsed gaining orbital energy. This perturbation effect is translated into a net inward shift in the node of the perturbed meteoroids. Such geometry with Jupiter occurred in 2004 over a meteoroid trail ejected by this comet during the 1862 A.D. return of the comet to perihelion. In order to study the predicted outburst produced by onerevolution meteoroids, the Spanish Photographic Meteor Network (SPMN) performed an extensive
\end{abstract}


campaign. As a part of this observational effort here are presented 10 accurate meteoroid orbits. We discuss their origin by comparing them with the theoretical orbital elements of the dust trails intercepting the Earth during the 2004 Perseid return.

Keywords: $109 \mathrm{P} /$ Swift-Tuttle, perseids, meteors, meteor outburst, dust trail

\section{Introduction}

The Perseid meteoroid stream produces yearly a meteor shower from late July to mid-August. The activity profile is more or less constant with a maximum Zenithal Hourly Rate (ZHR) of about 100 (Koschak and Rendtel, 1990; Jenniskens, 1994). However, associated with the return of the parent body comet 109P/Swift-Tuttle (Kosai et al., 1992) several meteor outbursts with ZHR over 300 were recorded between 1990 and 1994 (Brown and Rendtel, 1996; Jenniskens et al., 1998). Last passage by the perihelion of this comet in 1992 was also the occasion to study its dynamics. From these data Yau et al. (1994) obtained the orbital elements at each perihelion return of this Halley-type comet for more than 2000 years. Based on this work, it is possible to perform the prediction of Perseid meteor showers, from the method developed by Lyytinen and Van Flandern (2000), or Vaubaillon et al. (2005). The long-term dynamics of the Perseid meteoroid stream was already studied by Brown and Jones (1998), who demonstrated the stability of the orbital elements. Here, we compare the predicted orbital elements of the 2004 Perseid meteoroids with these obtained from accurate multiplestation observations. This way, not only the approaches can be proved, but our knowledge of the meteoroid stream is refined (Jenniskens, 1998). In this work we present the orbital elements of several bright Perseid meteors recorded from Spain in basis of multiple station photographic, video, and CCD observations.

\section{Instrumentation, Data Reduction and Observation Sites}

Perseid meteors were recorded by several professional and amateur stations participating in the framework of the SPMN. The campaign was made during the night of August 11-12 2002 under excellent sky conditions. Two professional video CCD stations working in common fields were established in Sierra Nevada and La Sagra Observatories (IAA) from which were obtained continuously images in order to derive the velocity and timing of the meteors. One additional professional station was operating in La Mayora (IAA-CSIC) by using a slow-scan CCD all sky camera prototype used for the development of the Spanish Fireball Network (Trigo-Rodríguez et al., 2005a). Additionally, several amateur stations were working in small camera projects in Castilla-La Mancha, Catalonia and Valencian regions. 
The geographic location and height of all stations that provided multistation meteor data are compiled in Table I. Amateur stations were composed by small camera batteries or slow-scan CCD detectors with $50-35 \mathrm{~mm}$ optics. The amateur stations were equipped with rotating shutters with accurately controlled frequency in order to estimate the geocentric velocity of the meteors. The part of the network operating in Andalucia recorded the time of appearance of the meteors from Sierra Nevada and La Sagra video records. In other regions, camera operators took time exposures with an accuracy of one second, while the time of occurrence of the bright meteors were taken from simultaneous visual observations.

The photographic negatives were developed and scanned at 2700 points per inch using a Kodak SprintScan scanner. Slow-scan CCD images were directly measured from the processed digital images. We used PhotoFinish 4 software to obtain the astrometry of the stars and the meteors. The astrometric measurements were then introduced into our Network software, which provides the equatorial coordinates of the meteors with an astrometric accuracy of $0.005^{\circ}$ for meteor photographs (additional details in TrigoRodríguez et al., 2004). This software also allows us to predict the position of every meteor from each station by assuming the typical values of ablation height. A search on the database for meteors appeared during the same observing interval in those positions makes easy the identification of the common multiple-station meteors. Once identified, the software estimates the atmospheric trajectory and radiant for each meteor. From the astrometric measurements of the shutter breaks and the trajectory length the velocity of the meteoroid was derived. An averaged value of observed velocities for each shutter-break was assumed as the preatmospheric velocity $V_{\infty}$ since the final deceleration is barely measurable for most cases. Finally, in order to determine orbital elements from our trajectory data we used the Meteoroid Orbit (MORB) program provided by Ceplecha et al. (2000) from the Ondrejov Observatory in the Czech Republic.

TABLE I

Observing stations

\begin{tabular}{lllll}
\hline Station (province) & Longitude & Latitude & Height $(\mathrm{m})$ & Observing technique \\
\hline Aspe (Alicante) & $0^{\circ} 47^{\prime} 44^{\prime \prime} \mathrm{W}$ & $38^{\circ} 20^{\prime} 31^{\prime \prime} \mathrm{N}$ & 275 & Camera battery + shutter \\
Bonilla (Cuenca) & $2^{\circ} 31^{\prime} 59^{\prime \prime} \mathrm{W}$ & $40^{\circ} 11^{\prime} 05^{\prime \prime} \mathrm{N}$ & 906 & Camera battery + shutter \\
Castelldefels (Barcelona) & $1^{\circ} 34^{\prime} 48^{\prime \prime} \mathrm{E}$ & $41^{\circ} 09^{\prime} 36^{\prime \prime} \mathrm{N}$ & 110 & Camera battery + shutter \\
Gualba (Barcelona) & $2^{\circ} 31^{\prime} 43^{\prime \prime} \mathrm{E}$ & $41^{\circ} 42^{\prime} 54^{\prime \prime} \mathrm{N}$ & 300 & Slow-scan CCD + shutter \\
La Mayora (Málaga) & $4^{\circ} 02^{\prime} 40^{\prime \prime} \mathrm{W}$ & $36^{\circ} 45^{\prime} 35^{\prime \prime} \mathrm{N}$ & 200 & Slow-scan CCD \\
La Sagra (Granada) & $2^{\circ} 33^{\prime} 53^{\prime \prime} \mathrm{W}$ & $37^{\circ} 58^{\prime} 53^{\prime \prime} \mathrm{N}$ & 1530 & Video CCD \\
Sierra Nevada (Granada) & $3^{\circ} 23^{\prime} 05^{\prime \prime} \mathrm{W}$ & $37^{\circ} 03^{\prime} 51^{\prime \prime} \mathrm{N}$ & 2896 & Video CCD \\
Titaigües (Valencia) & $1^{\circ} 04^{\prime} 59^{\prime \prime} \mathrm{W}$ & $39^{\circ} 51^{\prime} 52^{\prime \prime} \mathrm{N}$ & 906 & Camera battery + shutter \\
\hline
\end{tabular}




\section{Observations: Trajectory, Radiant and Orbital Data}

The observations were systematically programmed in order to maximize the common atmospheric volume recorded by the different instruments. We used the program Photographic centers for multiple station meteor observations in the same way as was explained in Trigo-Rodríguez et al. (2004). By knowing the geographical coordinates of the participating stations and the expected geometry of the meteor appearance from the radiant position, the common centers for each station were derived. In total, on August 11-12th during $6 \mathrm{~h}$ of multiple station work, around 300 meteors were imaged from the different stations. A great display of bright bolides occurred when the Earth crossed the dust trail. Figure 1 shows all fireballs recorded from La Sagra video CCD station in the interval $1 \mathrm{~h} 30 \mathrm{~min}$ and $2 \mathrm{~h} 30 \mathrm{~min}$ UT corresponding to Solar Longitude $=139.65^{\circ}$ (Trigo-Rodríguez et al., 2005b). Figure 2 shows as example the apparent radiant deduced from astrometrically reduced meteors. Most of them were recorded only from single stations and, in consequence, it was not possible to derive trajectory and radiant data. At this time, 21 double-station meteors have been clearly identified by our software, but only 10 were imaged using a rotating shutter and had convergence angles greater than $20^{\circ}$. The trajectory data of these accurately reduced meteors are given in Table II, which shows the code system used for identification, the apparent visual magnitude $\left(m_{\mathrm{v}}\right)$, the meteor trail beginning and end height on the

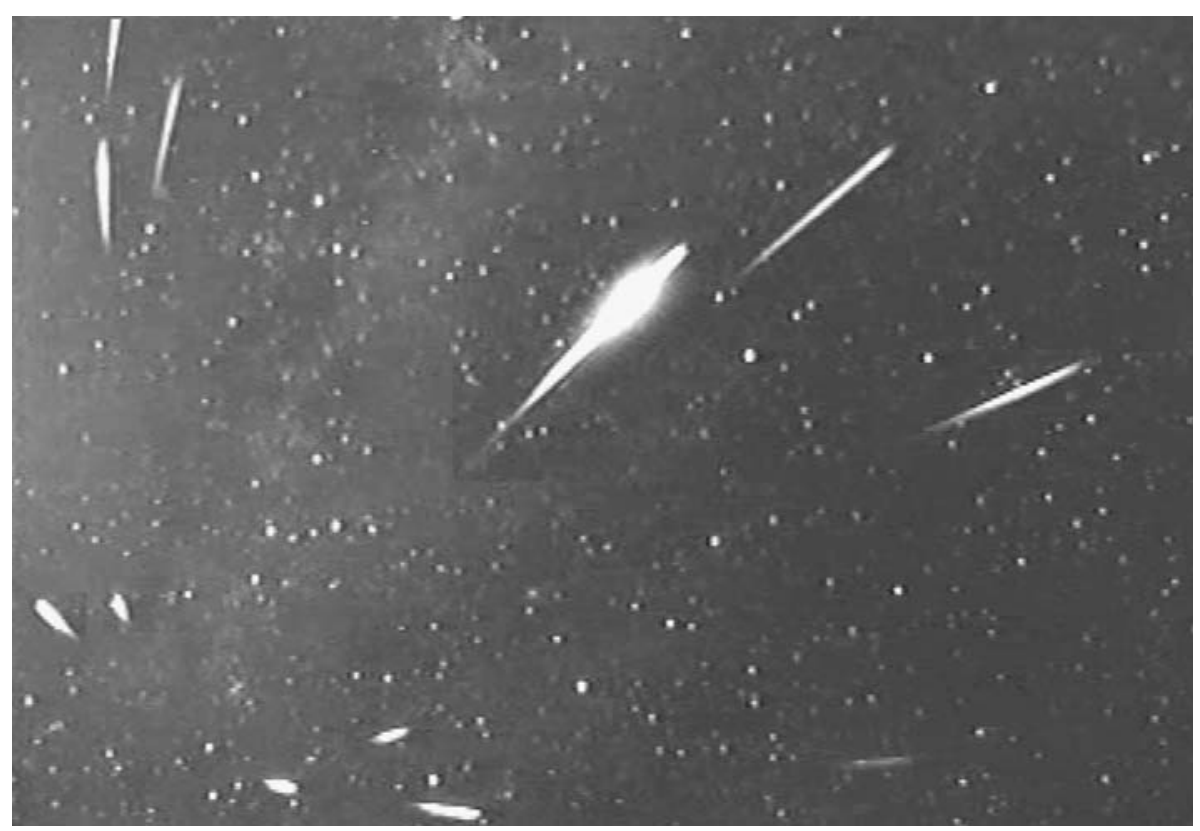

Figure 1. Fireballs recorded from La Sagra video CCD station. For more details see the text. 


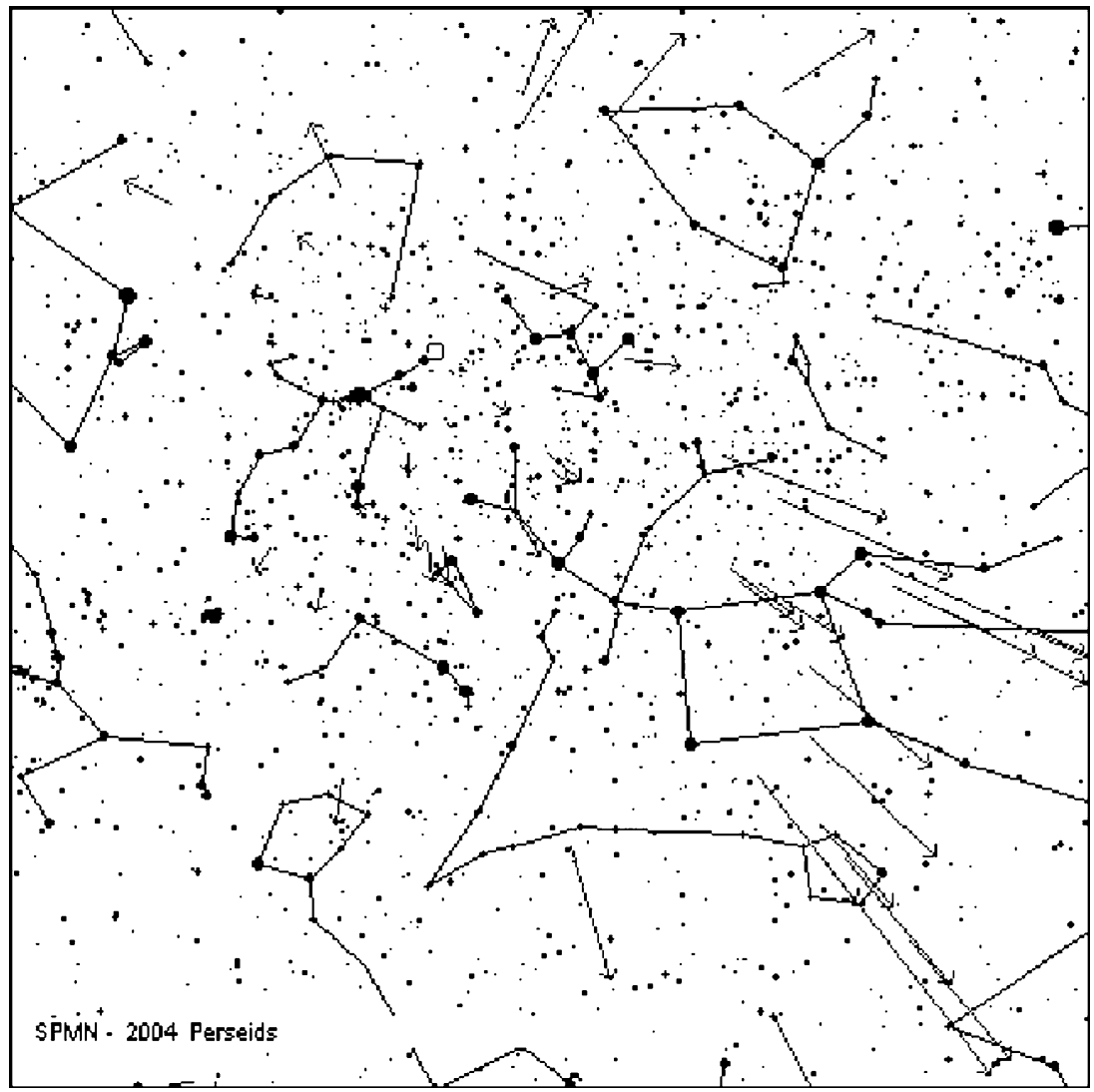

Figure 2. Perseid apparent radiant for all astrometrically reduced meteors. The deduced radiant appears plotted as a small square.

Earth's surface $\left(H_{\mathrm{b}}\right.$ and $H_{\mathrm{e}}$ in $\left.\mathrm{km}\right)$, the geocentric radiant coordinates $\left(\alpha_{\mathrm{g}}\right.$ and $\delta_{\mathrm{g}}$ to $\mathrm{Eq} 2000.0$ ) and the velocity in $\mathrm{km} / \mathrm{s}$ (at the top of atmosphere, geocentric and heliocentric). The apparent magnitude of the meteors was obtained by plotting the measured peak signal as function of the magnitude for different stars and for the meteors. Taking into account the different angular velocity of the meteors compared to the stars in the field is possible to estimate their apparent magnitude.

From the radiant position, apparition time and velocities estimated for the Perseid meteors listed in Table II we derived the orbital elements shown in Table III. For comparison the last row shows the computed ("theoretical") orbital elements for members of the 1862 and 826 dust trails meteoroids. The circumstances of the encounter of the Earth and the 1862 and 826 trails are plotted in Figure 3. The line represents the path of the Earth, while each dot represents the node of particles released by comet 109P/Swift-Tuttle during 
J.M ${ }^{\mathrm{a}}$. TRIGO-RODRIGUEZ ET AL.

TABLE II

Trajectory and radiant data

\begin{tabular}{llllllllll}
\hline Code & $m_{\mathrm{v}}$ & $\begin{array}{l}H_{\mathrm{b}} \\
(\mathrm{km})\end{array}$ & $\begin{array}{l}H_{\max } \\
(\mathrm{km})\end{array}$ & $\begin{array}{l}H_{\mathrm{e}} \\
(\mathrm{km})\end{array}$ & $\alpha_{\mathrm{g}}\left(^{\circ}\right)$ & $\delta_{\mathrm{g}}\left(^{\circ}\right)$ & $\begin{array}{l}V_{\infty} \\
(\mathrm{km} / \mathrm{s})\end{array}$ & $\begin{array}{l}V_{\mathrm{g}} \\
(\mathrm{km} / \mathrm{s})\end{array}$ & $\begin{array}{l}V_{\mathrm{h}} \\
(\mathrm{km} / \mathrm{s})\end{array}$ \\
\hline P1 & -8 & 123.9 & - & 86.1 & $45.61 \pm 0.02$ & $56.53 \pm 0.03$ & $60.7 \pm 0.2$ & 59.5 & 41.5 \\
P2 & -3 & 118.2 & 102.3 & 88.5 & $44.66 \pm 0.03$ & $57.81 \pm 0.02$ & $60.7 \pm 0.2$ & 59.5 & 41.5 \\
P3 & -4 & 108.2 & 101.2 & 96.5 & $44.82 \pm 0.02$ & $58.29 \pm 0.02$ & $60.4 \pm 0.2$ & 59.2 & 41.4 \\
P4 & -2 & 102.5 & - & 97.3 & $46.10 \pm 0.04$ & $57.63 \pm 0.04$ & $60.7 \pm 0.2$ & 59.4 & 41.5 \\
P5 & -3 & 115.9 & - & 80.9 & $45.71 \pm 0.04$ & $57.65 \pm 0.04$ & $60.6 \pm 0.2$ & 59.4 & 41.4 \\
P6 & -2 & 113.4 & 102.1 & 95.4 & $44.8 \pm 0.1$ & $57.8 \pm 0.1$ & $60.6 \pm 0.2$ & 59.4 & 41.4 \\
P7 & -3 & 111.9 & 102.1 & 94.1 & $47.1 \pm 0.1$ & $57.3 \pm 0.1$ & $60.8 \pm 0.2$ & 59.6 & 41.4 \\
P8 & 0 & 109.2 & 101.5 & 96.0 & $46.8 \pm 0.1$ & $56.5 \pm 0.1$ & $60.3 \pm 0.2$ & 59.1 & 41.3 \\
P9 & -3 & 113.2 & 98.6 & 89.5 & $46.7 \pm 0.1$ & $57.2 \pm 0.1$ & $60.0 \pm 0.2$ & 58.5 & 41.4 \\
P10 & -5 & 114.3 & 102.2 & 90.3 & $44.0 \pm 0.2$ & $57.5 \pm 0.2$ & $60.6 \pm 0.2$ & 59.4 & 41.4 \\
Average & - & - & - & - & $45.6 \pm 0.1$ & $57.4 \pm 0.1$ & $60.5 \pm 0.2$ & 59.3 & 41.4 \\
\hline
\end{tabular}

its return to perihelion in 1862 and 826 . From the nodes, was forecasted a Perseid outburst associated with the 1862 dust trail on August 11, 2004 around $21 \mathrm{~h}$ UT. However, a minor contribution of the 826 dust trail meteoroids cannot be ruled out.

\section{Discussion}

The geocentric radiant was computed from 239 simulated particles found in the very vicinity of the Earth in August 2005. Its theoretical position for the outburst Perseid meteoroids at solar longitude $139.5^{\circ}$ is $\mathrm{RA}=45.7^{\circ}$ and $\mathrm{DEC}=57.6^{\circ}$ (Equinox 2000.0) derived by using Neslusan et al. (1998) software under the assumption that the particles were released in 1862 . The averaged observational radiant for the 10 double-station meteoroids (last row in Table II) fits well the predicted position. Those values seem distinguishable from the radiant of the annual stream members located at $\mathrm{RA}=44.7^{\circ}$ and $\mathrm{DEC}=56.9^{\circ}$. In general, bright outburst Perseid meteors have orbits exhibiting lower dispersion than those expected for those associated with the annual stream. This fact translates in a very tight radiant for the outburst meteoroids as was found by Jenniskens et al. (1998).

The theoretical orbital elements are given as separate in Table III, for the trails 1862 and 826 . To some degree the orbital values are also dependent on the space-criterion used to select the simulated particles. This is especially true to the semi-major axis value. Slightly tighter criterion was used than in the previous more general radiant derivation. The wider criterion is especially 
ORBITAL ELEMENTS OF 2004 PERSEID METEOROIDS

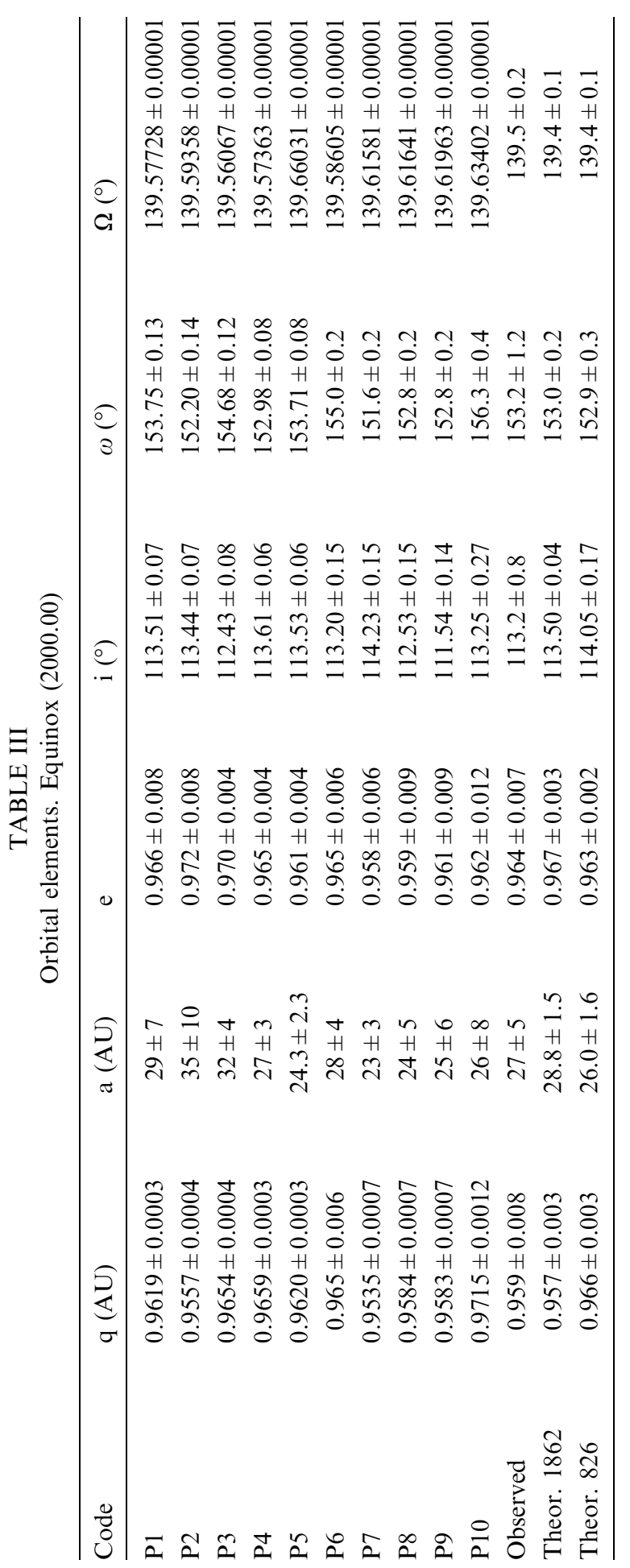




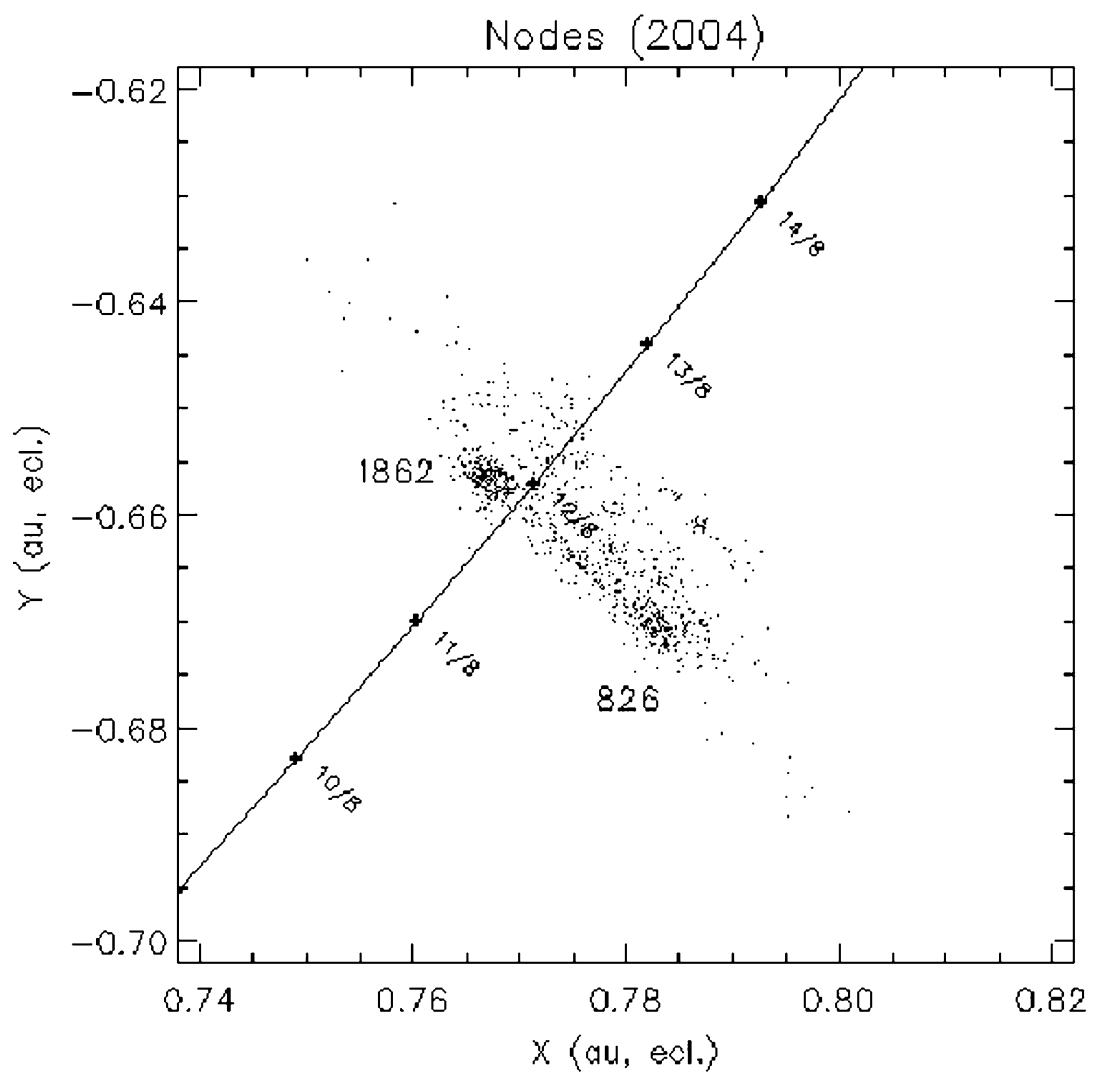

Figure 3. Circumstances of the encounter of the Earth and the 1862 and 826 dust trails. For more details see the text.

suitable in concluding if an outburst may happen or not. The tighter criterion is probably better to get the best theoretical values for the elements.

The discrepancy in the two radiants (observed vs. theoretical) suggests that not only meteoroids from the 1862 dust trail were recorded during the SPMN campaign. Of course, a small background contribution of the annual shower or from the 826 dust trail cannot be ruled out. However, we must say that the annual activity during the observational period was about three times lower than that associated with the outburst (Arlt, 2004). In order to study other possible dust trail contributions we took the orbital solution of comet 109P/Swift-Tuttle to simulate the evolution of older dust trails, ejected back in year 59 A.D. Due to the lack of observations before the 19th century, these elements are uncertain. However, it appears than a trail ejected in 826 A.D. could be also contributing to the 2004 outburst. Additional orbital data would allow to answer the level of its contribution to the activity in the observed period. 
By comparing the theoretical and observational orbital data given in Table III is clear that the 2004 Perseid outburst was mainly produced by the interception of the dust trail released from 109P/Swift-Tuttle comet during its 1862 return to perihelion. As was forecasted, in order to make possible the interception of this dust trail by the Earth in 2004 is required a gravitational impulse of the planet Jupiter. Consequently, although such gravitational perturbation was necessarily present, the orbital elements are not clearly different from those of other Perseid returns (see e.g., Jenniskens et al., 1998).

\section{Conclusions}

The 2004 Perseid outburst monitored by the SPMN was caused by the 1862 dust trail of 109P/Swift-Tuttle, such as was forecasted by Lyytinen \& Vaubaillon's works. The acquired data enabled us to compare the results from the model and the reality. The averaged radiant data and orbital elements match very well, inside the observational uncertainty. However, the derived scattering of the radiant cannot be due solely to observational error and it is possible that an un-modeled stream component was active during the time of our observations. It is not only explained by possible presence of background meteoroids from the annual stream because a further analysis enabled us to identify the 826 A.D. trail as peaking at the same time as the 1862. However, from the lower spatial density expected for an older 826 trail, it seems likely that its contribution was lower than this one produced by the one-revolution 1862 trail.

\section{References}

Arlt, R.: 2004, Perseid 2004, International Meteor Organization Report: http://www.imo.net/ node $/ 610$.

Brown, P. and Rendtel, J.: 1996, Icarus 124, 414-428.

Brown, P. and Jones, J.: 1998, Icarus 133, 36-68.

Ceplecha, Z., Spurny, P., and Borovička, J.: 2000. MORB Software to Determine Meteoroid Orbits, Ondrejov Observatory, Czech Republic.

Jenniskens, P.: 1994, A\&A 287, 990-1013.

Jenniskens, P.: 1998, Earth Planets Space 50, 555-567.

Jenniskens, P., Betlem, H., de Lignie, M., ter Kuile, C., van Vliet, M. C. A., van't Leven, J., Koop, M., Morales, E., and Rice, T.: 1998, MNRAS 301, 941-954.

Kosai, H., Kiuchi, T., Tatum, J. B., Sugie, A., Nakano, S., Scotti, J. V., and Marsden, B. G.: 1992, IAU Circular $\mathbf{5 6 2 0 .}$

Koschack, R. and Rendtel, J.: 1990, WGN 18, 119-131.

Lyytinen, E. and Van Flandern, T.: 2000, Earth Moon Planets 82/83, 149-166.

Neslusan, L., Svoren, J., and Porubcan, V.: 1998, A\&A 331, 411-413. 


\section{J.M . TRIGO-RODRIGUEZ ET AL.}

Trigo-Rodríguez, J. M., Llorca, J., Lyytinen, E., Ortiz, J. L., Sánchez Caso, A., Pineda, C., and Torrell, S.: 2004, Icarus 171, 219-228.

Trigo-Rodríguez, J. M., Castro-Tirado, A., Llorca, J., Fabregat, J., Martínez, V. J., Reglero, V., Jelínek, M., Kubánek, P., Mateo, T. and de Ugarte Postigo, A.: 2005a, Earth Moon Planets online first.

Trigo-Rodríguez, J. M., Castro-Tirado, A., Jelinek M. and 18 coauthors.: 2005b, WGN 33, 5-8.

Vaubaillon, J., Colas, F., and Jorda, F.: 2005, $A \& A$ 439, 751-760.

Yau, K., Yeomans, D., and Weissman, P.: 1994, MNRAS 266, 305-316. 